\title{
Isobolographic additivity among lacosamide, lamotrigine and phenobarbital in a mouse tonic-clonic seizure model
}

\author{
Maria W. Kondrat-Wróbel ${ }^{1, B-D}$, Jarogniew J. Łuszzzki ${ }^{1,2, A, C-F}$ \\ ${ }^{1}$ Department of Pathophysiology, Medical University of Lublin, Poland \\ ${ }^{2}$ Isobolographic Analysis Laboratory, Institute of Rural Health, Lublin, Poland \\ A - research concept and design; $\mathrm{B}$ - collection and/or assembly of data; $\mathrm{C}$ - data analysis and interpretation; \\ $D$ - writing the article; $E$ - critical revision of the article; $F$ - final approval of the article
}

\section{Address for correspondence \\ Jarogniew J. Łuszczki}

E-mail: jarogniew.luszzzki@umlub.pl

\section{Funding sources}

None declared

\section{Conflict of interest}

Prof. J.J. Łuszzzki has been involved in the design and development of new antiepileptics and CNS drugs. He has received, within the last 5 years, an unrestricted research grant from GlaxoSmithKline (Brentford, UK). Dr. M.W. Kondrat-Wróbel has no conflict of interest to disclose.

Acknowledgements

This study was supported by grants (DS474/20122014 and DS474/2015-2016) from the Medical University of Lublin (Poland).

Received on July 14, 2016

Reviewed on December 28, 2016

Accepted on February 23, 2017

\section{Abstract}

Background. Epilepsy is a serious neurological disease affecting about 1\% of people worldwide (65 million). Seizures are controllable with antiepileptic drugs (AEDs) in about 70\% of epilepsy patients, however, there remains about $30 \%$ of patients inadequately medicated with these AEDs, who need a satisfactory control of their seizure attacks. For these patients, one of the treatment options is administration of 2 or 3 AEDs in combination.

Objectives. To determine the anticonvulsant effects of a combination of 3 selected AEDs (i.e., lacosamide - LCM, lamotrigine - LTG and phenobarbital - PB) at the fixed-ratio of 1:1:1 in a mouse maximal electroshock-induced (tonic-clonic) seizure model by using isobolographic analysis.

Material and methods. Seizure activity was evoked in adult male albino Swiss mice by a current (sinewave, $25 \mathrm{~mA}, 500 \mathrm{~V}, 50 \mathrm{~Hz}, 0.2$ s stimulus duration) delivered via auricular electrodes. Type I isobolographic analysis was used to detect interaction for the 3-drug combination.

Results. With type I isobolographic analysis, the combination of LCM, LTG and PB (at the fixed-ratio of 1:1:1) exerted additive interaction in the mouse maximal electroshock-induced (tonic-clonic) seizure model.

Conclusions. The combination of LCM with LTG and PB produced additive interaction in the mouse tonicclonic seizure model, despite various molecular mechanisms of action of the tested AEDs.

Key words: antiepileptic drugs, isobolographic analysis, maximal electroshock, 3-drug combination

DOI

10.17219/acem/69132

Copyright

Copyright by Author(s)

This is an article distributed under the terms of the

Creative Commons Attribution Non-Commercial License

(http://creativecommons.org/licenses/by-nc-nd/4.0/) 


\section{Introduction}

Pharmacotherapy in epilepsy is still a challenge for clinicians and epileptologists because about one third of patients with epilepsy are not sufficiently treated with licensed antiepileptic drugs (AEDs) in monotherapy. ${ }^{1}$ To reduce both seizure frequency and seizure activity in patients with refractory epilepsy, some combinations of 2 or 3 various AEDs should be considered as the most appropriate treatment regimen. ${ }^{2-5}$ Nowadays, some combinations of AEDs are recommended to refractory patients in 3 consecutive monotherapies with the established first-line AEDs. ${ }^{2,3}$ Generally, the combined application of AEDs possessing different anticonvulsant modes of action is clinically recommended to potentiate the anticonvulsive properties of AEDs. ${ }^{5,6}$

Direct clinical assessment of the interactions among 3 drugs in epilepsy patients is extremely difficult due to ethical restrictions and methodological limitations. However, preclinical studies in animal in vivo models of epilepsy provide us with plausible proof on the accurate type of interactions occurring among the selected 3 AEDs. There is no doubt that in preclinical in vivo experiments based on isobolographic analysis of interaction, it is easily to confirm whether the theoretically selected AED combinations are beneficial or not. ${ }^{7}$ This is the reason to conduct experiments on animals with isobolography and subsequently translate the most favorable combinations into clinical settings.

Lacosamide (LCM) belongs to the $3^{\text {rd }}$ generation of AEDs. Its unique molecular mechanisms of anticonvulsant action rely mainly on the enhancement of slow inactivation in voltage-gated sodium channels, preventing the sodium channels from opening and terminating the action potentials in hyperactive (epileptically changed) neurons for an extended time. ${ }^{8,9}$ Thus, LCM inhibits repetitive neuronal firing and stabilizes hyperexcitable neuronal membranes without affecting the physiological functions of the neurons. ${ }^{8,9}$ At present, LCM is licensed as an added drug prescribed to adult patients with partial-onset seizures that undergo generalization. ${ }^{10,11}$

The combined administration of LCM with lamotrigine (LTG) and phenobarbital (PB), 2 commonly prescribed AEDs for patients suffering from tonic-clonic and partial onset seizures, ${ }^{12}$ should produce a favorable 3 -drug combination, offering protection from tonic-clonic seizures in patients with epilepsy who are resistant to monotherapeutic use of these AEDs. ${ }^{13}$ Furthermore, the combined treatment with LCM, LTG and PB theoretically fulfils the general principles of a perfect AED combination, giving a maximum of therapeutic effect and a minimum of side effects, due to diverse molecular mechanisms of action. ${ }^{6}$ However, this hypothetically ideal combination requires experimental confirmation in a maximal electroshockinduced seizure (MES) test in mice. Of note, the MES test is considered an animal test reflecting generalized tonicclonic seizures and partial convulsions in humans. ${ }^{14}$
This study was aimed at characterizing the interactions among LCM, LTG and PB in the MES test in mice. We used type I isobolographic analysis to evaluate the interaction among the fully effective drugs (i.e., LCM, LTG and PB) because this method is designed to investigate interaction in preclinical experiments. ${ }^{15}$

\section{Material and methods}

In all experimental tests, we used 4-month-old male albino Swiss mice. The local Ethics Committee (University of Life Sciences, Lublin, Poland) approved all of the described protocols and procedures (License No. 45/2014).

LCM (UCB Pharmaceuticals, Brussels, Belgium), LTG (GlaxoSmithKline, Brentford, UK) and PB (Polfa, Warszawa, Poland) were dispersed in an aqueous solution of Tween 80 (Sigma-Aldrich, St. Louis, USA). The AEDs were injected intraperitoneally (i.p.) to animals at the pretreatment times as follows: LCM - $30 \mathrm{~min}$, LTG and PB - $60 \mathrm{~min}$, respectively.

Alternating current ( $25 \mathrm{~mA}, 0.2 \mathrm{~s}$ stimulus duration) generated by rodent shocker (Hugo Sachs Elektronik, March, Germany) was used to evoke maximal electroconvulsions (seizure activity) in mice, as described earlier. ${ }^{16,17}$ The animals, after receiving different drug doses, were subjected to the MES test and protection from maximal electroconvulsions was noted so as to construct dose-effect functions for the investigated AEDs (LCM, LTG and PB) when injected separately, as described earlier. ${ }^{16,18}$ The anticonvulsant properties of LCM, LTG and PB, when injected alone and combined at a fixed-ratio of 1:1:1, were presented in the form of median effective doses (i.e., $\mathrm{ED}_{50}$ and $\mathrm{ED}_{50 \text { exp }}$ values).

Type I isobolographic analysis assessed the nature of the interactions among the 3 AEDs in combination. ${ }^{15,19}$ Of note, isobolography is a mathematical method that makes it possible to precisely determine the kind of interactions occurring when drugs are combined together. Isobolographic analysis distinguishes 5 classes of drug interactions: synergy (supra-additivity), additivity, indifference, relative antagonism (sub-additivity), and absolute antagonism (infra-additivity). ${ }^{20,21}$ Subsequently, a linear regression analysis based on logarithms and probits made it possible to construct dose-effect functions for the studied AEDs (LCM, LTG and PB). ${ }^{22}$ To establish the proper class of interactions, the investigated AEDs are usually administered together in fixed drug dose ratio combinations. However, to properly select the fixed-ratio combinations, one should verify whether the dose-effect functions of the studied AEDs if used alone are collateral. If the drugs have their dose-response effect curves parallel to each other, at least 3 fixed-ratios (i.e., 3:1, 1:1 and $1: 3$ ) are experimentally investigated with isobolography. ${ }^{23}$ Of note, many more fixed-ratio combinations are sometimes required to establish the exact types of interactions for particular drug mixtures. ${ }^{21}$ On the other hand, for drugs 


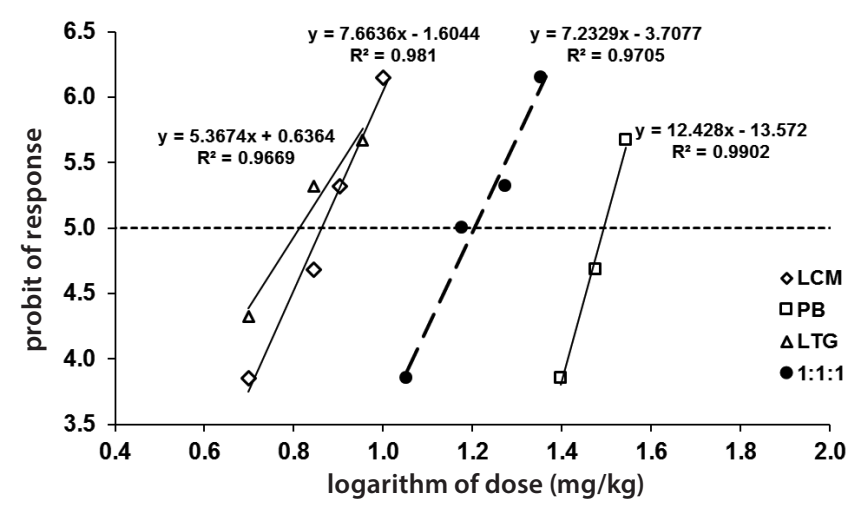

Fig. 1. Dose-effect functions of lacosamide, lamotrigine and phenobarbital injected separately and in combination (1:1:1) against maximal electroconvulsions in mice

Equations for the anticonvulsant effects of LCM, LTG, PB and their combination are presented graphically; $\mathrm{R}^{2}$ - coefficient of determination.

having their dose-effect functions mutually nonparallel, only a fixed-ratio of 1:1 is analyzed with isobolography. ${ }^{24}$ To the best of our knowledge, calculations of doses for particular drugs with their nonparallel dose-effect functions, applied in fixed-ratio combinations more diverse than 1:1 (i.e., 1:3 and 3:1), are not precise because the effects produced by the drug doses may be either over- or underestimated, ultimately providing improper information on the interaction among the drugs. ${ }^{15,24}$ The isobolographic rule, concerning parallelism of dose-response effect curves for the tested AEDs when they are used alone, is obligatory not only for 2-drug combinations, but also for 3- and 4-drug combinations. ${ }^{24}$ After verifying the parallelism of dose-effect functions of LCM, LTG and PB, the median additive dose of the mixture of these 3 AEDs (ED 50 add $)$ at a fixed-ratio combination of 1:1:1 was calculated according to the methods described earlier. ${ }^{16,17}$ Admittedly, LCM displayed parallelism to PB and LTG. However, these latter 2 AEDs, when compared to each other, had their doseeffect functions nonparallel. Therefore, only 1 fixed-ratio (1:1:1) underwent investigation in this study because, as mentioned earlier, other tested fixed-ratios (i.e., 1:1:3, 1:3:1, 3:1:1, 3:3:1, 3:1:3 or 1:3:3) could provide false classification of interaction. ${ }^{15,24-26}$ The anticonvulsant protection offered by the 3 AEDs in mixture 1:1:1 was presented in the form of $\mathrm{ED}_{50 \text { exp }}$ value, reflecting a dose of 3 drugs in mixture that protected $50 \%$ of the mice from MES-induced seizures. ${ }^{16,17}$
The unpaired Student's t-test was used to statistically compare the $\mathrm{ED}_{50 \text { exp }}$ and $\mathrm{ED}_{50 \text { add }}$ values.

\section{Results}

LCM, LTG and PB injected separately protected the mice from maximal electroconvulsions. From log-probit equations of dose-effect functions, the $\mathrm{ED}_{50}$ values for LCM, LTG and PB were calculated (Fig. 1), amounting to $7.27 \pm 0.77 \mathrm{mg} / \mathrm{kg}$ (LCM), $6.50 \pm 0.80 \mathrm{mg} / \mathrm{kg}$ (LTG) and $31.21 \pm 2.04 \mathrm{mg} / \mathrm{kg}(\mathrm{PB})$, respectively (Table 1 ).

Parallelism of dose-effect functions was observed between LCM and LTG, as well as between LCM and PB (Table 1, Fig. 1). In contrast, dose-effect functions between LTG and PB were non-collateral (Table 1).

LCM, PB and LTG combined together at a fixed-ratio of 1:1:1 produced an anti-electroshock effect and the $\mathrm{ED}_{50 \text { exp }}$ value was $15.99 \pm 1.80 \mathrm{mg} / \mathrm{kg}$. With type I isobolographic analysis, the mixture of LCM, LTG and PB at a fixed-ratio of 1:1:1 produced additivity in mice subjected to the MES test (Fig. $2 \mathrm{~A}-\mathrm{C}$ ). The $\mathrm{ED}_{50 \text { exp }}$ value for the mixture of LCM, LTG and PB was $15.99 \pm 1.80 \mathrm{mg} / \mathrm{kg}$ and displayed no significant difference from the $\mathrm{ED}_{50 \text { add }}$ value, which amounted to $14.99 \pm 0.71 \mathrm{mg} / \mathrm{kg}$.

Neither LCM, LTG and PB administered singly (in doses reflecting their $\mathrm{ED}_{50}$ values from the maximal electroconvulsions), nor the combination of LCM, LTG and PB (1:1:1) disturbed memory processes in mice challenged with the passive avoidance task, changed skeletal muscular strength in mice in the grip-strength test, and impaired motor coordination in mice subjected to the chimney test (Table 2).

\section{Discussion}

It was found experimentally that the combination of LCM, LTG and PB (1:1:1) produced additivity in the mouse MESinduced seizure test. The illustrated additivity for the combination of the 3 AEDs (LCM, LTG and PB) can be compared to the effects reported from experiments conducted for the combinations of 2 AEDs (i.e., PB + LTG, LCM + LTG, and $\mathrm{LCM}+\mathrm{PB}$ ) in the same seizure test. Previously, we have reported supra-additivity (synergy) for the combination of PB with LTG at a fixed-ratio of 1:1 against

Table 1. Anticonvulsant properties of lamotrigine, lacosamide and phenobarbital when administered separately in the MES-induced seizure test in mice

\begin{tabular}{|l|c|c|c|}
\hline \multicolumn{1}{|c|}{ Drug } & $\mathrm{ED}_{50}$ & $\mathrm{n}$ & Combination \\
\hline LTG & $6.50 \pm 0.80$ & 24 & LCM vs LTG \\
\hline LCM & $7.27 \pm 0.77$ & 16 & parallelism \\
\hline PB & $31.21 \pm 2.04$ & 16 & pCM vs PB \\
\hline
\end{tabular}

Results are median effective doses ( $E_{50}$ values $[\mathrm{mg} / \mathrm{kg}] \pm$ SEM, where SEM is standard error of the mean) of LCM, LTG and PB administered separately in the MES-induced seizure test in mice. The drugs were administered systemically (i.p.), as follows: LCM - 30 min, LTG and PB - 60 min before the MES-induced seizures; $n$ - total number of animals used at doses whose expected anticonvulsant effects ranged between the $4^{\text {th }}$ and $6^{\text {th }}$ probit; ${ }^{\#}$ test for parallelism was performed according to Litchfield and Wilcoxon. ${ }^{22}$ For more details see Fig. 1. 
Table 2. Effects of lamotrigine, lacosamide and phenobarbital, administered alone and in mixture at a fixed-ratio of 1:1:1 on long-term memory in the passive avoidance task, muscular strength in the grip-strength test and motor performance in the chimney test in mice

\begin{tabular}{|c|c|c|c|}
\hline Treatment [mg/kg] & Retention time $[\mathrm{s}]$ & Muscular strength $[\mathrm{N}]$ & Motor coordination impairment [\%] \\
\hline Vehicle + vehicle & $180(180 ; 180)$ & $0.987 \pm 0.067$ & 0 \\
\hline LTG (6.50) + vehicle & $180(180 ; 180)$ & $0.948 \pm 0.062$ & 0 \\
\hline LCM (7.27) + vehicle & $180(180 ; 180)$ & $0.949 \pm 0.065$ & 0 \\
\hline PB (31.21) + vehicle & $180(180 ; 180)$ & $0.962 \pm 0.072$ & 0 \\
\hline LCM (2.58) + LTG (2.31) + PB (11.09) & $180(180 ; 180)$ & $0.952 \pm 0.071$ & 0 \\
\hline
\end{tabular}

Results are median retention times (with $25^{\text {th }}$ and $75^{\text {th }}$ percentiles in parentheses) from the passive avoidance task, mean muscular strengths \pm SEM from the grip-strength test and percentage of animals with impairment of motor coordination from the chimney test. Each experimental group consisted of 8 mice. Doses of particular drugs (in parentheses) correspond to their $\mathrm{ED}_{50}$ values from the MES test.
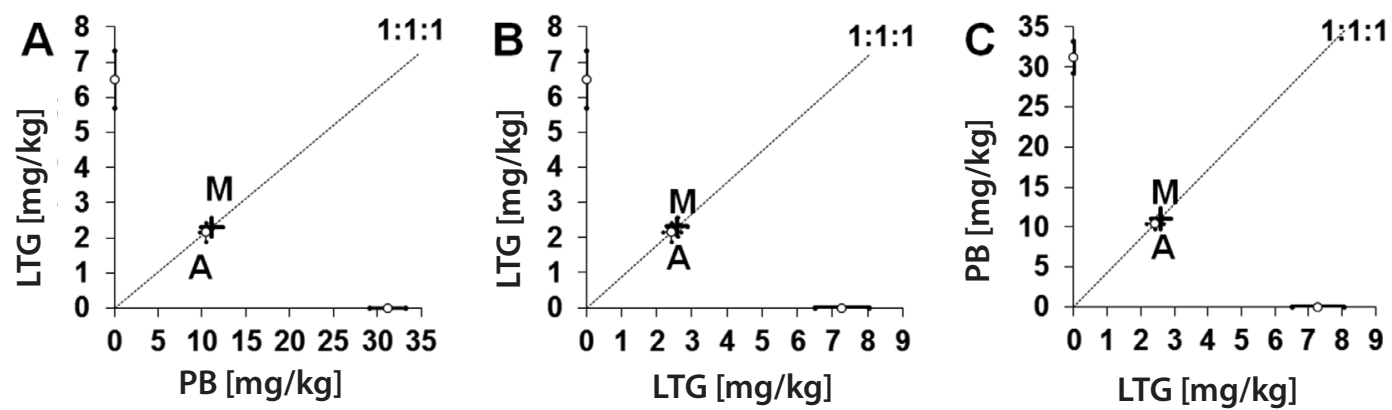

Fig. 2 A-C. Isobolograms illustrating additivity among lacosamide, lamotrigine and phenobarbital in mice subjected to maximal electroconvulsions

The $\mathrm{ED}_{50}$ exp and $\mathrm{ED}_{50}$ add values \pm SEM for the mixture of LCM, LTG and PB (1:1:1) were plotted graphically as points M and A. A - additive interaction of PB with LTG; $B$ - additive interaction of LCM with LTG; C - additive interaction of LCM with PB.

maximal electroconvulsions in mice. ${ }^{27}$ Unfortunately, no information is available on the type of interactions for the combinations of LCM with PB and LTG against maximal electroconvulsions in mice. Nevertheless, in another experimental model of electrically-induced seizures, a $6 \mathrm{~Hz}$ psychomotor seizure test, the combination of LCM with LTG also produced supra-additivity (synergy) in mice. ${ }^{28}$

The interaction among the 3 AEDs (LCM, LTG and PB) from this study can also be compared to the interactions observed previously for the combinations of LCM, carbamazepine $(\mathrm{CBZ})$ and $\mathrm{PB},{ }^{16}$ or topiramate (TPM), $\mathrm{CBZ}$ and $\mathrm{PB}$, against maximal electroconvulsions in mice. ${ }^{17}$ More specifically, a supra-additivity (synergy) for the combination of TPM, CBZ and PB was found in the MES test in mice. ${ }^{17}$ In contrast, the replacement of TPM with LCM in the mixture of LCM, CBZ and PB resulted in additivity with a trend to sub-additivity in the MES test in mice. ${ }^{16}$ On the other hand, the replacement of CBZ with LTG produced additivity without any trend to sub-additivity (antagonism) in the MES test, as documented in this study for LCM, LTG and PB. Thus, it can be concluded that the mixture of LCM, LTG and PB is more favorable than that of LCM, CBZ and PB and, simultaneously, less beneficial than that of CBZ, PB and TPM in the MES-induced seizure test.

From a theoretical viewpoint, the mixture of LCM, LTG and $\mathrm{PB}$ should be favorable and of particular relevance for epileptic patients because of the diverse mechanisms of action of the component drugs. ${ }^{6}$ Of note, the conjunction of LCM, LTG and PB complies with theoretical presumptions related to combining drugs with diverse mechanisms of anticonvulsant action. As is widely known, LTG attaches to inactive voltage-dependent sodium channels and thus, it reduces the sustained repetitive firing in neurons. ${ }^{29}$ LTG also blocks voltage-activated $\mathrm{N}$ - and P/Q-type calcium channels. ${ }^{30}$ LCM selectively enhances slow inactivation of sodium channels and makes hyperexcitable neurons stable. ${ }^{8,31}$ Additionally, LCM allosterically blocks NMDA receptors containing the $\mathrm{NR} 2 \mathrm{~B}$ subunit. ${ }^{8,31}$ As regards $\mathrm{PB}$, the drug enhances the effects of $\mathrm{GABA}_{\mathrm{A}}$ receptors by attaching to an allosteric regulatory site within the complex of $\mathrm{GABA}_{\mathrm{A}}$-receptor and chloride ionophore. ${ }^{32-34}$ Furthermore, PB inhibits AMPA receptors. ${ }^{35}$ Thus, taking into account the mentioned mechanisms of action of LCM, LTG and $\mathrm{PB}$, the mixture of these AEDs should supra-additively inhibit generalized tonic-clonic seizures in mice challenged with maximal electroconvulsions because their mechanisms of anticonvulsant action complement each other. Unfortunately, in this study we barely found additivity among LCM, LTG and PB against maximal electroconvulsions in mice.

Much more attention should be paid to the doses of particular AEDs used - we combined LCM, LTG and PB in doses 3 times lower than their respective $\mathrm{ED}_{50}$ values as denoted singly in the MES test in mice. This is the reason 
we obtained results reflecting monotherapy with one effective AED, as regards the inhibition of maximal electroconvulsions in mice. Of note, any decrease in drug doses is beneficial due to the limitation of acute adverse effects exerted by AEDs used separately that would occur if the AEDs could be injected in higher doses into the mice.

Here we also determined the potential of acute adverse effects evoked by LCM, LTG and PB in a mixture with respect to their influence on motor performance, memory processes and skeletal muscular strength in the animals subjected to the chimney, passive avoidance and grip-strength tests, respectively. Generally, drugs or their mixtures that significantly affect motor performance in animals disturb coordination or make the animals unable to climb backward up the plastic Plexiglas tube within 60 s. ${ }^{36,37}$ Similarly, in the passive avoidance test, drugs or their mixtures that significantly affect the animals' long-term memory may impair memory acquisition and/or remembering in experimental animals, which manifests by the entrance of the mice into the dark box of the passive avoidance apparatus without a period of staying in the light compartment for up to $180 \mathrm{s.}{ }^{38,39}$ In the grip-strength test, drugs or their mixtures that significantly alleviate skeletal muscular strength induce flaccidity of the animal's bodies or reduce muscle tension in the mice that manifest in a low force in grasping and pulling the squared stainless steel wire mesh connected to the electronic dynamometer. ${ }^{40,41}$ Of note, the mixture of LCM, LTG and PB was administered to animals in doses corresponding to the $\mathrm{ED}_{50 \text { exp }}$ value obtained in the MES-induced seizure test. In this study, the mixture of LCM, LTG and PB had no significant impact on motor performance in mice since all of the tested animals climbed backward up the tube within 1 min (Table 2). Similarly, the AEDs in combination had no significant effect on memory processes in the animals tested - the mice remained in the light compartment of the apparatus for up to 3 min (Table 2). The combination of LCM, LTG and PB had no significant impact on the animals' skeletal muscular strength because no significant changes in grasping and pulling the wire mesh connected to the dynamometer were observed in the mice (Table 2). Considering the results from these 3 behavioral tests, it can be concluded that LCM, LTG and PB in combination (1:1:1) exerted no acute side effects as compared to the animals from the control group, suggesting that the AEDs in mixture, at doses reflecting the $\mathrm{ED}_{50 \text { exp }}$ from the MES test, were safe enough to be recommended for application in further clinical settings, especially in epileptic patients treated with these AEDs in monotherapy.

Summing up, it can be concluded that the combined application of LCM, LTG and PB exerted additivity against maximal electroconvulsions in mice. Finally, LCM combined with LTG and PB can modify the interaction among drugs from synergistic to additive, despite their various anticonvulsant modes of action.

\section{References}

1. Kwan P, Schachter SC, Brodie MJ. Drug-resistant epilepsy. N Engl J Med. 2011;365:919-926.

2. Stephen LJ, Brodie MJ. Seizure freedom with more than one antiepileptic drug. Seizure. 2002;11:349-351.

3. Stephen LJ, Forsyth M, Kelly K, Brodie MJ. Antiepileptic drug combinations - Have newer agents altered clinical outcomes? Epilepsy Res. 2012;98:194-198.

4. Barker-Haliski M, Sills GJ, White HS. What are the arguments for and against rational therapy for epilepsy? Adv Exp Med Biol. 2014;813: 295-308.

5. Perucca E. Pharmacological principles as a basis for polytherapy. Acta Neurol Scand Suppl. 1995;162:31-34.

6. Deckers CL, Czuczwar SJ, Hekster YA, et al. Selection of antiepileptic drug polytherapy based on mechanisms of action: The evidence reviewed. Epilepsia. 2000;41:1364-1374.

7. Luszczki JJ, Czuczwar SJ. Preclinical profile of combinations of some second-generation antiepileptic drugs: An isobolographic analysis. Epilepsia. 2004;45:895-907.

8. Errington AC, Stohr T, Heers C, Lees G. The investigational anticonvulsant lacosamide selectively enhances slow inactivation of voltagegated sodium channels. Mol Pharmacol. 2008;73:157-169.

9. Rogawski MA, Tofighy A, White HS, Matagne A, Wolff C. Current understanding of the mechanism of action of the antiepileptic drug lacosamide. Epilepsy Res. 2015;110:189-205.

10. Biton V, Gil-Nagel A, Isojarvi J, Doty P, Hebert D, Fountain NB. Safety and tolerability of lacosamide as adjunctive therapy for adults with partial-onset seizures: Analysis of data pooled from three randomized, double-blind, placebo-controlled clinical trials. Epilepsy Behav. 2015;52:119-127.

11. Zadeh WW, Escartin A, Byrnes W, et al. Efficacy and safety of lacosamide as first add-on or later adjunctive treatment for uncontrolled partialonset seizures: A multicentre open-label trial. Seizure. 2015;31: 72-79.

12. Glauser T, Ben-Menachem E, Bourgeois B, et al. Updated ILAE evidence review of antiepileptic drug efficacy and effectiveness as initial monotherapy for epileptic seizures and syndromes. Epilepsia. 2013;54:551-563.

13. Loscher W. Single versus combinatorial therapies in status epilepticus: Novel data from preclinical models. Epilepsy Behav. 2015;49: 20-25.

14. Loscher W, Fassbender CP, Nolting B. The role of technical, biological and pharmacological factors in the laboratory evaluation of anticonvulsant drugs. II. Maximal electroshock seizure models. Epilepsy Res. 1991;8:79-94.

15. Tallarida RJ. Revisiting the isobole and related quantitative methods for assessing drug synergism. J Pharmacol Exp Ther. 2012;342:2-8.

16. Kondrat-Wróbel MW, Łuszczki JJ. Interaction of three-drug combination of lacosamide, carbamazepine and phenobarbital in the mouse maximal electroshock-induced seizure model - An isobolographic analysis. Health Prob Civil. 2016;10(1):55-61.

17. Luszczki JJ. Isobolographic analysis of interaction for three-drug combination of carbamazepine, phenobarbital and topiramate in the mouse maximal electroshock-induced seizure model. Pharmacology. 2016;97:259-264.

18. Żółkowska D, Zagaja M, Miziak B, et al. Isobolographic assessment of interactions between retigabine and phenytoin in the mouse maximal electroshock-induced seizure model and chimney test. Health Prob Civil. 2016;10(4):54-59.

19. Loewe $S$. The problem of synergism and antagonism of combined drugs. Arzneimittelforschung. 1953;3:285-290.

20. Luszczki JJ, Borowicz KK, Swiader M, Czuczwar SJ. Interactions between oxcarbazepine and conventional antiepileptic drugs in the maximal electroshock test in mice: An isobolographic analysis. Epilepsia. 2003;44:489-499.

21. Luszczki JJ, Czuczwar SJ. Isobolographic and subthreshold methods in the detection of interactions between oxcarbazepine and conventional antiepileptics - A comparative study. Epilepsy Res. 2003;56: 27-42.

22. Litchfield JT, Wilcoxon F. A simplified method of evaluating doseeffect experiments. J Pharmacol Exp Ther. 1949;96:99-113.

23. Luszczki JJ, Wu JZ, Raszewski G, Czuczwar SJ. Isobolographic characterization of interactions of retigabine with carbamazepine, lamotrigine, and valproate in the mouse maximal electroshockinduced seizure model. Naunyn Schmiedebergs Arch Pharmacol. 2009;379:163-179. 
24. Luszczki JJ. Isobolographic analysis of interaction between drugs with nonparallel dose-response relationship curves: A practical application. Naunyn Schmiedebergs Arch Pharmacol. 2007;375:105-114.

25. Tallarida RJ. Quantitative methods for assessing drug synergism. Genes Cancer. 2011;2:1003-1008.

26. Luszczki JJ, Czuczwar SJ. Biphasic characteristic of interactions between stiripentol and carbamazepine in the mouse maximal electroshock-induced seizure model: A three-dimensional isobolographic analysis. Naunyn Schmiedebergs Arch Pharmacol. 2006;374:51-64.

27. Luszczki JJ, Czuczwar M, Kis J, et al. Interactions of lamotrigine with topiramate and first-generation antiepileptic drugs in the maximal electroshock test in mice: An isobolographic analysis. Epilepsia. 2003;44:1003-1013.

28. Shandra A, Shandra P, Kaschenko O, Matagne A, Stohr T. Synergism of lacosamide with established antiepileptic drugs in the $6-\mathrm{Hz}$ seizure model in mice. Epilepsia. 2013;54:1167-1175.

29. Nakatani $\mathrm{Y}$, Masuko $\mathrm{H}$, Amano T. Effect of lamotrigine on $\mathrm{Na}(\mathrm{v}) 1.4$ voltage-gated sodium channels. J Pharmacol Sci. 2013;123:203-206.

30. Pisani A, Bonsi P, Martella G, et al. Intracellular calcium increase in epileptiform activity: Modulation by levetiracetam and lamotrigine. Epilepsia. 2004;45:719-728.

31. Stohr T, Kupferberg HJ, Stables JP, et al. Lacosamide, a novel anticonvulsant drug, shows efficacy with a wide safety margin in rodent models for epilepsy. Epilepsy Res. 2007;74:147-154.

32. Mathers DA, Wan X, Puil E. Barbiturate activation and modulation of GABA(A) receptors in neocortex. Neuropharmacology. 2007;52: 1160-1168.

33. Twyman RE, Rogers CJ, Macdonald RL. Differential regulation of gamma-aminobutyric acid receptor channels by diazepam and phenobarbital. Ann Neurol. 1989;25:213-220.
34. Rho JM, Donevan SD, Rogawski MA. Direct activation of GABA $A_{A}$ receptors by barbiturates in cultured rat hippocampal neurons. J Physiol. 1996:497(2):509-522.

35. Ko GY, Brown-Croyts LM, Teyler TJ. The effects of anticonvulsant drugs on NMDA-EPSP, AMPA-EPSP, and GABA-IPSP in the rat hippocampus. Brain Res Bull. 1997;42:297-302.

36. Boissier JR, Tardy J, Diverres JC. A new simple method to explore the "tranquillizing" action: The chimney test [in French]. Pharmacology. 1960;3:81-84.

37. Luszczki JJ, Czernecki R, Wojtal K, Borowicz KK, Czuczwar SJ. Agmatine enhances the anticonvulsant action of phenobarbital and valproate in the mouse maximal electroshock seizure model. J Neural Transm. 2008;115:1485-1494.

38. Venault P, Chapouthier G, de Carvalho LP, et al. Benzodiazepine impairs and beta-carboline enhances performance in learning and memory tasks. Nature. 1986;321:864-866.

39. Luszczki JJ, Wojcik-Cwikla J, Andres MM, Czuczwar SJ. Pharmacological and behavioral characteristics of interactions between vigabatrin and conventional antiepileptic drugs in pentylenetetrazole-induced seizures in mice: An isobolographic analysis. Neuropsychopharmacology. 2005;30:958-973.

40. Meyer OA, Tilson HA, Byrd WC, Riley MT. A method for the routine assessment of fore- and hindlimb grip strength of rats and mice. Neurobehav Toxicol. 1979;1;233-236.

41. Nieoczym D, Luszczki JJ, Czuczwar SJ, Wlaz P. Effect of sildenafil on the anticonvulsant action of classical and second-generation antiepileptic drugs in maximal electroshock-induced seizures in mice. Epilepsia. 2010;51:1552-1559. 